\title{
Exatidão máxima no cálculo da probabilidade com distribuição Normal da Pressão Arterial Diastólica
}

\author{
$\underline{\text { Maurício D .C Balboni }}^{1 *} \quad$ Lucas M. Tortelli $^{1} \quad$ Alice F. Finger $^{2} \quad$ Aline B. Loreto $^{2}$ \\ Universidade Federal de Pelotas \\ ${ }^{1}$ Curso Ciência da Computação/CDTec \\ ${ }^{2}$ Programa de Pós-graduação em Computação/CDTec \\ CEP 96001-970, Campus Porto, Pelotas-RS \\ E-mail: baalbis@gmail.com, \{lmtortelli, affinger, aline.loreto@inf.ufpel.edu.br $\}$
}

\section{RESUMO}

No estudo das variáveis aleatórias contínuas sobre o conjunto dos números reais, $\mathbb{R}$, um dos problemas é o cálculo de probabilidades, visto que é necessário resolver uma integral definida da função densidade que, na maioria das vezes, não possui primitiva explícita ou cuja primitiva não é simples de se obter. A função densidade de probabilidade com distribuição Normal não possui primitiva explícita, neste caso métodos de integração numérica podem ser empregados para calcular integrais e gerar tabelas de probabilidade [6].

A análise intervalar surgiu com o objetivo de diminuir erros numéricos gerados em procedimentos computacionais. Na matemática intervalar o valor real x é aproximado por um intervalo $\mathrm{X}$ que possui limites inferior $(\underline{x})$ e superior $(\bar{x})$, de forma que o intervalo contenha $\mathrm{x}$ [5].

O presente trabalho tem como objetivo calcular a probabilidade com distribuição Normal com entradas reais através do Método 1/3 de Simpson e com entradas intervalares aplicando o Método de Simpson Intervalar definido por Caprani et al. [2].

A distribuição Normal é uma das mais importantes distribuições contínuas de probabilidade, pois muitos fenômenos aleatórios comportam-se de maneira similar a essa distribuição. Possui os parâmetros $\mu$ e $\sigma^{2}$ referentes a média e variância, respectivamente.

Uma aplicação importante para a probabilidade com distribuição Normal, por exemplo, é o cálculo da probabilidade de uma população estar entre os limites aceitáveis(abaixo de $80 \mathrm{mmHg}$ ) da pressão sanguínea diastólica.

A pressão arterial consiste na força por unidade de área (tendo $\mathrm{mmHg}$ como unidade de medida) que o próprio sangue, depois de bombeado pelo músculo cardíaco, exerce sobre as paredes dos vasos sanguíneos enquanto percorre cada milímetro do corpo, garantindo assim que todo ele receberá o sangue. Essa pressão é medida em dois tipos, a pressão arterial sistólica e a pressão arterial diastólica devido a pressão arterial sofrer algumas oscilações ao longo do seu caminho por todo o corpo [4].

Na pressão arterial diastólica normalmente o valor é conhecido como a pressão arterial mínima, correspondente ao momento em que o ventrículo esquerdo volta a encher-se para retomar todo o processo da circulação. Essa pressão é considerada alta se estiver a maior parte do tempo acima de $90 \mathrm{mmHg}$ e é considerada normal se estiver a maior parte do tempo abaixo de $80 \mathrm{mmHg}$ [4].

Por exemplo, deseja-se calcular qual a probabilidade de que uma mulher adulta tenha pressão sanguínea diastólica entre 30 e $60 \mathrm{mmHg}, 60$ e $90 \mathrm{mmHg}$ e 90 até $120 \mathrm{mmHg}$, sabendo que a pressão sanguínea de mulheres entre 18 e 74 anos é normalmente distribuída com média $77 \mathrm{mmHg}$ e desvio padrão de 11,6 mmHg. Considerando $\delta=0,01$ de precisão para os limites do intervalo $X=[x-\delta, x+\delta]$ e utilizando um sistema de ponto flutuante $\mathrm{F}(10,3,-10,10)$ (ou com três casas decimais) tem-se os seguintes resultados na Tabela 1.

*bolsista de Iniciação Científica PBIC/UFPel 
Tabela 1: Resultados reais e intervalares para a pressão diastólica

\begin{tabular}{c|c|c}
\hline Exemplo & Pn & Pni \\
\hline $30 \mathrm{mmHg}$ e $60 \mathrm{mmHg}$ & 0,071 & {$[0,061 ; 0,081]$} \\
\hline $60 \mathrm{mmHg}$ e $90 \mathrm{mmHg}$ & 0,797 & {$[0,787 ; 0,807]$} \\
\hline $90 \mathrm{mmHg}$ e $120 \mathrm{mmHg}$ & 0,131 & {$[0,121 ; 0,141]$} \\
\hline
\end{tabular}

Observa-se que os valores da probabilidade real $P n$ estão contidos na probabilidade intervalar Pni, $P n \subset P n i$, refletindo em uma solução intervalar que contém os erros numéricos gerados em procedimentos computacionais. A fim de analisar a qualidade do intervalo solução $X=P n i$, utilizam-se as seguintes estimativas para o erro [5]:

Erro Absoluto $(E A):|x-m(X)|<w(X) / 2$, onde $m(X)$ é o ponto médio do intervalo $\mathrm{X}$ e $w(X)=|\bar{x}-\underline{x}|$ é o diâmetro do intervalo $\mathrm{X}$;

Erro Relativo $(E R):\left|\frac{x-m(X)}{x}\right| \leq \frac{w(X)}{2 \min |X|}$ se $0 \notin X$, onde $|X|=\{|X|: x \in X\}$.

Tabela 2: Erros Absoluto e Relativo dos intervalos

\begin{tabular}{c|c|c}
\hline Exemplo & EA & ER \\
\hline $30 \mathrm{mmHg}$ e $60 \mathrm{mmHg}$ & $1,38777878078 \times 10^{-17}<0,01$ & $1,9546180011 \times 10^{-16} \leq 0,16393442623$ \\
\hline $60 \mathrm{mmHg}$ e $90 \mathrm{mmHg}$ & $0,0<0,01$ & $0,0 \leq 0,012706480305$ \\
\hline $90 \mathrm{mmHg}$ e $120 \mathrm{mmHg}$ & $0,0<0,01$ & $0,0 \leq 0,0826446280992$ \\
\hline
\end{tabular}

Com base nas análises de qualidade do intervalo na Tabela 2, verifica-se que a utilização da probabilidade intervalar apresentou uma exatidão maior, minimizando os erros gerados pela probabilidade real, possibilitando a utilização da probabilidade intervalar em diversas aplicações de forma mais confiável. Um dos problemas de saúde atualmente é a pressão arterial, e verificar a probabilidade de uma população estar entre os limites aceitáveis (abaixo de $80 \mathrm{mmHg}$ ) da pressão sanguínea diastólica é fundamental para que os órgãos de saúde adotem estratégias para tratamento e prevenção de problemas cardíacos, entre outros.

A linguagem utilizada para obtenção dos resultados foi Python, os critérios para a escolha da linguagem da biblioteca são encontrados em Balboni et al. [1]. Detalhes dos algoritmos intervalares estão disponíveis em Finger [3]. O computador utilizado possui as seguintes configurações: processador Intel Core i5 CPU 760 @ 2.80GHz Quad-Core, L1 Cache 64Kb, L2 Cache 512Kb, L3 Cache 8Mb, Memória RAM de 16GB DDR3 1333MHz, armazenamento HD Sata 500GB modelo ATA Samsung HD502HJ, sistema operacional Linux Ubuntu 13.10.

Palavras-chave: Distribuição Normal, Pressão arterial, Probabilidade Intervalar

\section{Referências}

[1] Balboni, M. D. C.; Tortelli, L. M.; Lorini, M.; Furlan, V. S.; Finger, A. F.; Loreto, A. B, "Critérios para Análise e Escolha de Ambientes Intervalares". Revista Jr de Iniciação Científica em Ciências Exatas e Engenharia, Rio Grande - RS, n.7, 2014.

[2] Caprani, O.; Madsen, K.; Nielsen, H. B, "Introduction to interval analysis". IMM - Informatics and Mathematical Modelling, 2002.

[3] Finger, A. F, "Extensão Intervalar para as Variáveis Aleatórias com Distribuições Uniforme, Normal, Gama, Exponencial e Pareto", Dissertação de Mestrado, Universidade Federal de Pelotas, 2014.

[4] Medida Correta da Pressão Arterial, Disponível em: http://www.eerp.usp.br/ope/manual.htm. Acessado em 10 de fevereiro de 2014.

[5] Ratschek, H.; Rokne, R, “New Computer Methods for Global Optimization”, Ellis Horwood, 1988.

[6] Santos, M. G, "Probabilidades Autovalidáveis para as Variáveis Aleatórias Exponencial, Normal e Uniforme”, Tese de Doutorado, Universidade Federal de Pernambuco, Recife, 2010. 